\title{
Investigation of Degradation Mechanism of Rabeprazole with Solid State Pharmaceutical Excipients
}

\author{
Shan Ren ${ }^{1,3}$, Thao Truong-Dinh Tran ${ }^{1}$, Phuong Ha-Lien Tran ${ }^{1}$, Yun-Seok Rhee ${ }^{2}$ and Beom-Jin Lee ${ }^{1 \dagger}$ \\ ${ }^{1}$ Bioavailability Control Laboratory, College of Pharmacy, Kangwon National University, Chuncheon 200-701, Korea \\ ${ }^{2}$ School of Pharmacy, Sungkyunkwan University, Suwon, Gyeonggi-do 440-746, Korea \\ ${ }^{3}$ Department of Pharmaceutics and Analytical Chemistry, Faculty of Pharmaceutical Sciences, \\ University of Copenhagen, Copenhagen, DENMARK
}

(Received August 7, 2010 • Revised November 26, 2010 • Accepted November 27, 2010)

\begin{abstract}
Rabeprazole sodium (RPN) is known to be very unstable at acidic condition or some acidic pharmaceutical excipients such as acrylic acid polymer (carbomer 934) with carboxylic acids. Thus, degradation mechanism of binary blends of rabeprazole with pharmaceutical excipients in a solid state without using solvents at three different ratios $(3: 1,1: 1$ and 1:3) was investigated using Fourier transform infrad (FTIR) spectroscopy. Alkalizer (MgO), neutral hydroxypropymethylcellulose (HPMC 4000) were also tested for comparison. The binary blends were stored under accelerated conditions $\left(40^{\circ} \mathrm{C} / 75 \%\right.$ relative humidity) for two weeks. The concentration of thioether rabeprazole from the binary blends with acidic carbomer 934 increased as the rabeprazole concentration decreased. In addition, the degradation half-life of rabeprazole as well as the relative peak area ratios obtained from FTIR spectra of $\mathrm{S}=\mathrm{O}$ stretching at $1094.1 \mathrm{~cm}^{-1}$ decreased consistently as the fraction of carbomer 934 increased due to its sensitivity between the basic benzimidazole nitrogen and carboxylic acid group of carbomer 934. The physical appearance also turned into strong brown color in the presence of carbomer 934. In contrast, there were no significant changes in the degradation kinetics of rabeprazole with MgO and HPMC 4000 in a solid state. This present study demonstrated that the solid-state compatibility test with the aid of HPLC chromatographic and FTIR spectral analyses could offer a valuable methodology to select suitable pharmaceutical excipients and to elucidate the degradation mechanism of RPN for drug formulations at the early formulation stages.
\end{abstract}

Key words - Rabeprazole sodium, Thioether rabeprazole, Pharmaceutical excipients, Solid-state compatibility, Degradation mechanism

In the preparation of solid dosage formulations, it is essential to combine drugs with pharmaceutical excipients as adjuvants. Pharmaceutical excipients are known to be key components in pharmaceutical dosage forms having a great impact on in vitro release and in vivo bioavailability of drugs (Serajuddin et al., 1999; Byrn et al., 2001; Bruni et al., 2004; Cornaire et al., 2004; Wang et al., 2004). Most of all, pharmaceutical excipients can affect the physicochemical stability of drugs in dosage forms since they may function as stability enhancers or as promoters of the degradation of drugs (Loukas et al., 1998; Huang and Tong, 2004; Waterman and Adami, 2005; Urbanetz, 2006). Thus, rapid selection of pharmaceutical excipients for the evaluation of drug-excipient compatibility is essential at the early formulation approaches.

Rabeprazole sodium, a benzimidazole class of proton-pump inhibitors (PPIs) has been used recently worldwide for healing,

†Corresponding Author:

Tel : +82-33-250-6919, E-mail : beomjinlee@gmail.com

DOI : $10.4333 /$ KPS.2010.40.6.367 symptom relief and also for the prevention of relapse of acidpeptic diseases such as duodenal, gastric and esophageal ulceration (Carswell and Goa, 2001). Rabeprazole is labile to acids and moisture but relatively stable under alkaline conditions (El-Gindy et al., 2002). At acidic or neutral pH, rabeprazole is converted non-enzymatically primarily to thioether rabeprazole, resulting in the discoloration of drugs in solution as well as in solid-state (Richardson et al., 1998; Desta et al., 2002; Uno et al., 2005; Ren et al., 2008).

Due to the $\mathrm{pH}$ sensitivity of drug in solution and solid-state, the selection of optimal pharmaceutical excipients with different functional groups is crucial to maximize drug stability in the development of a stable dosage form of rabeprazole. Although the low stability of rabeprazole is reported under the acidic conditions, no detailed information on degradation behaviors of rabeprazole with $\mathrm{pH}$ dependent pharmaceutical excipients in a solid state is discussed yet.

The purpose of this work was to compare chemical stability of RPN in a solid state binary blend with pharmaceutical excipients during the storage under accelerated conditions 
$\left(40^{\circ} \mathrm{C} / 75 \%\right.$ relative humidity) for two weeks. Based on the acidity and alkalinity, three different excipients such as carbamer, $\mathrm{MgO}$ and HPMC were chosen. Acrylic acid polymer (carbomer 934) with carboxylic acids containing 56-68\% carboxylic acid $(\mathrm{COOH})$ groups in structures on the dry basis was chosen (Rowe et al., 2003). Alkaline $\mathrm{MgO}$ and neutral HPMC 4000 were also chosen for the comparison. Then, physicochemical properties of RPN such as drug content, physical appearance and FTIR spectra of drug in a solid state binary blend were characterized.

\section{Materials and methods}

\section{Materials}

Rabeprazole sodium was purchased from Satya Sai Residency (Hyderabad, India). Hypromellose (Metolose ${ }^{\circledR}$, HPMC 4000) was obtained from Shin-Etsu Chemical Co., Ltd. (Tokyo, Japan). $\mathrm{MgO}$ and diethylamine were purchased from Junsei Chemical Co., Ltd. (Tokyo, Japan). Carbomer 934 was obtained from Wonpoong Chemical Co. (Seoul, Korea). HPLCgrade methanol and acetonitrile were purchased from Fisher Scientific Korea Ltd. (Seoul, Korea). All other chemicals were of reagent grade and were used without further purification.

\section{Stability of binary blends}

Binary blends of rabeprazole with different pharmaceutical excipients were weighed directly in $2 \mathrm{~mL}$ vials and mixed on a micro-mixer (Taitec, Japan) for $30 \mathrm{~min}$. Each vial was sealed in a plastic bottle and stored at $40^{\circ} \mathrm{C}$ and $75 \%$ relative humidity (RH). Samples were collected and analyzed after storing for a designated time interval.

HPLC analysis of rabeprazole and its main degradation product

For the preparation of HPLC samples, $1 \mathrm{~mL}$ methanolic diethylamine $(0.1 \%)$ was immediately added to each vial to dissolve the contents and to prevent further degradation of rabeprazole. Subsequently the solution was transferred into a $100 \mathrm{~mL}$ volumetric flask and $0.1 \%$ methanolic diethylamine was added so that the final volume $100 \mathrm{~mL}$.

A reverse phase HPLC system was employed for quantification of rabeprazole and its corresponding degradation products. The HPLC system (Waters, USA) consisted of a pump (Waters ${ }^{\mathrm{TM}} 600$ Controller), an autosampler (Waters ${ }^{\mathrm{TM}}$ 717 plus Autosampler), a degasser (Waters ${ }^{\mathrm{TM}}$ In-line Degasser), an analytical column (Luna $5 \mu, \mathrm{C}_{18}, 4.6 \times 150 \mathrm{~mm}$ ) protected by a guard column (Gemini $\left.\mathrm{C}_{18}, 4 \times 3.0 \mathrm{~mm} \mathrm{ID}\right)$ and a UV-VIS spectrophotometric detector (Waters ${ }^{\mathrm{TM}} 486$ Tunable
Absorbance Detector) set at $284 \mathrm{~nm}$. The mobile phase was phosphate buffer ( $\mathrm{pH}$ 7.2), methanol, and acetonitrile at a ratio of 50:25:25 at a flow rate of $1.2 \mathrm{~mL} / \mathrm{min}$. The HPLC data processing was performed using Borwin ${ }^{\circledR} 1.20$ software.

\section{FTIR analysis}

ATR/FTIR spectra (Bio-Rad, Series UMA-500, USA) were recorded with the wavenumber range of $4000-400 \mathrm{~cm}^{-1}$ using a FTS 3000 MX Excalibur (USA) FTIR spectrometer. Complete microimplants could be investigated in a non-destructive manner using an attenuated total reflection (ATR) unit. Each spectrum was the average of 100 scans using a spectral resolution of $4 \mathrm{~cm}^{-1}$. FTIR spectra were recorded and stored using spectroscopic software (Merlin, Ver1.3).

\section{LC/MS identification of rabeprazole and its main degradation product}

The HPLC peak fraction of degradation product with 12.36 min retention time was applied into the LC/MS to identify the main degradation product of rabeprazole. The mass spectra were acquired using a Finnigan TSQ Quantum Ultra AM triple stage quadrupole mass spectrometer (Thermo, San Jose, CA, USA) equipped with an electrospray ionization (ESI) interface. The vaporizer temperature was set to $100^{\circ} \mathrm{C}$ and nitrogen was applied as the sheath gas. The heated capillary was maintained at $350^{\circ} \mathrm{C}$. Mass analysis was performed in the positive ion mode with the source current set at $5 \mathrm{~mA}$, and the potential of the tube lens was set at 82 volts. The $\mathrm{m} /$ $\mathrm{z}$ scanning ranged from 100 to 500. The type of column and composition of mobile phase were the same as described above for the HPLC analysis.

\section{SEM analysis}

The morphologies of the pure substance and binary blends were observed using a SEM (S-4300, Hitachi, Japan) at an acceleration voltage ranging from 10 to $15 \mathrm{kV}$ during scanning. Samples were prepared by dropping the microparticle suspension on a quartz wafer, air-drying overnight, and then sputtering with gold. Analyses of all samples were recorded at room temperature.

\section{Data processing}

The best-fit straight line was determined by the linear regression based on least squares method. The correlation of the curves was calculated. Data obtained from the experiments were subjected to an ANOVA test for statistical analysis. The $\mathrm{p}$ value $<0.05$ was considered statistically significant. 


\section{Results and Discussion}

\section{Effect of the types of pharmaceutical excipients}

The physicochemical nature of pharmaceutical excipients incorporated into drug-excipient blends can have a great impact on the stability of drugs (Serajuddin et al., 1999). Three different types of pharmaceutical excipients were chosen in this study in view of the $\mathrm{pH}$ related instability of rabeprazole.

Surface morphologies of rabeprazole and its binary blends with pharmaceutical excipients at a drug-excipient ratio of 1:3 examined by SEM are shown in Figure 1. Rabeprazole alone formed rectangular or irregular crystals while wrinkled surfaces of HPMC 4000 and spherical shells of $\mathrm{MgO}$ or carbomer 934 with clusters were observed. There were no significant changes of surface morphologies found during storage conditions.

The color change of each sample was observed after two weeks of storage under accelerated condition $\left(40^{\circ} \mathrm{C} / 75 \% \mathrm{RH}\right)$. A slight yellow coloration in rabeprazole alone, a strong brown coloration in binary blends containing carbomer 934 and no visible coloration in other binary blends with HPMC and $\mathrm{MgO}$ were observed after two weeks of storage, indicating instability of rabeprazole in the binary blends with carbomer 934 .

To understand the detailed information on chemical stability of rabeprazole, changes in the amount of rabeprazole in the absence and presence of pharmaceutical excipients at a drugexcipient ratio of $3: 1$ as a function of storage time were exam-
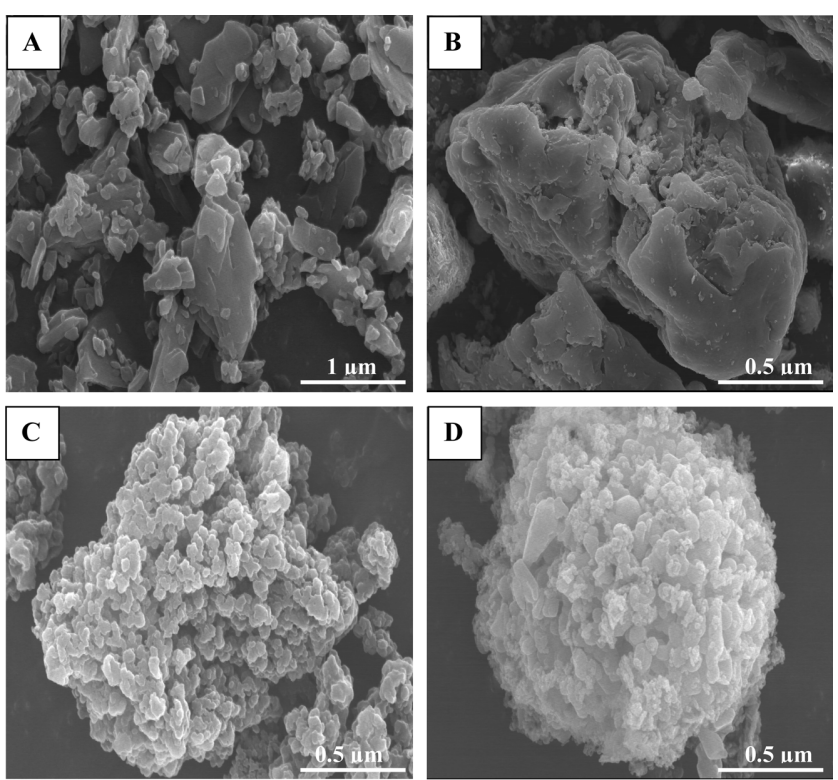

Figure 1. Surface morphologies by SEM of (A) rabeprazole alone and its binary blends with (B) HPMC 4000, (C) MgO and (D) carbomer 934 at a drug-excipient ratio of 1:3 after two week storage at $40^{\circ} \mathrm{C}$ and $75 \% \mathrm{RH}$. ined (Figure 2). Thioether rabeprazole was successfully separated as the main degradation product and identified by liquid chromatography mass spectroscopy (LC/MS) as described before (Ren et al., 2008). A statistically significant $(p<0.05)$ decrease in the amount of rabeprazole remaining was observed with carbomer 934 after one week $(82.1 \%)$ and two weeks $(56.8 \%)$ whereas no statistically significant change was found in other samples.

ATR/FTIR can be used as a method of choice to investigate the chemical stability of rabeprazole on a molecular level. Figure 3 gives FTIR spectra of rabeprazole alone and its binary

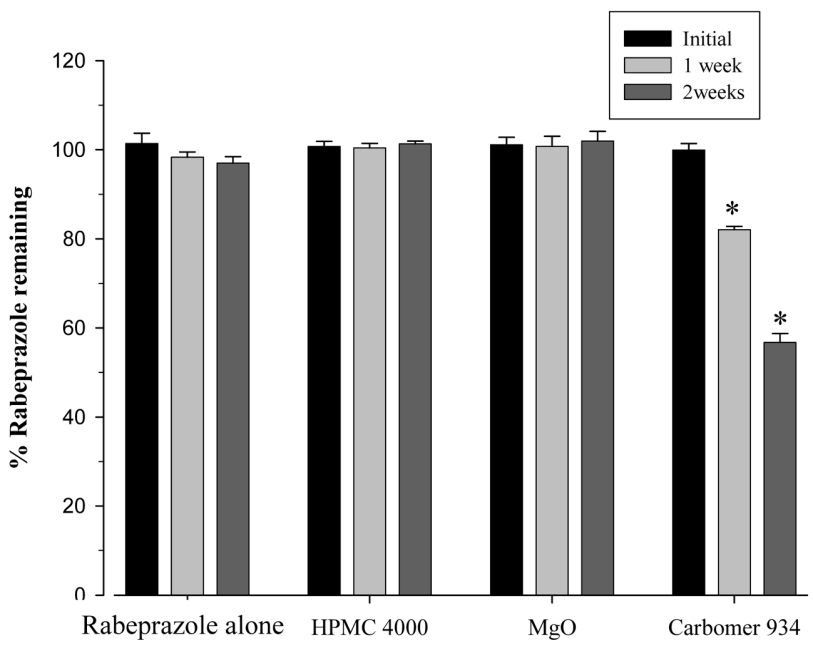

Figure 2. Changes in the amount of rabeprazole in the absence and presence of pharmaceutical excipients at a drug-excipient ratio of $3: 1$ as a function of storage time. ${ }^{*} \mathrm{p}<0.05$, significantly different from the amount at time zero (initial time).

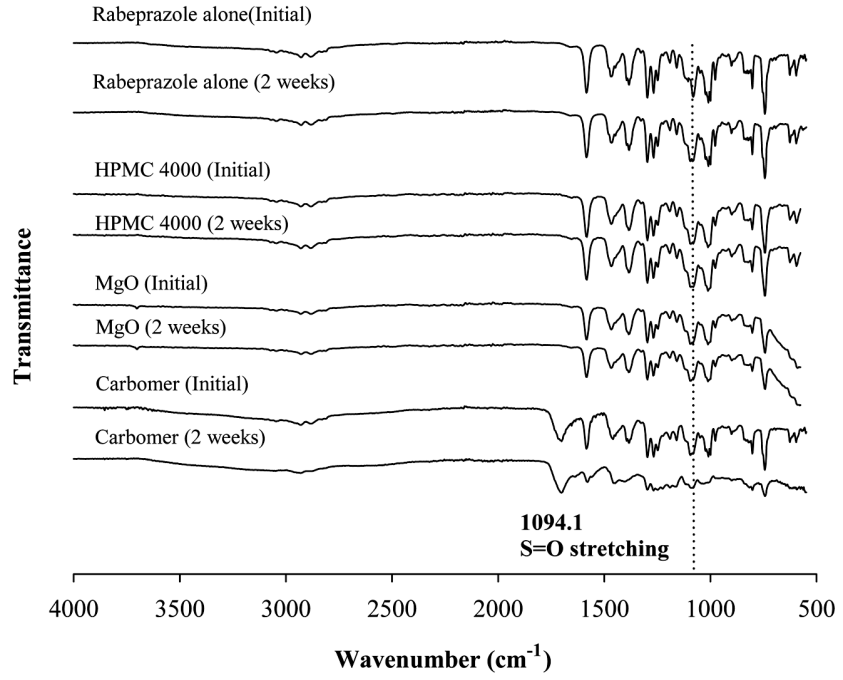

Figure 3. FTIR spectra of rabeprazole alone and its binary blends with HPMC 4000, $\mathrm{MgO}$ and carbomer 934 at a drug-excipient ratio of $3: 1$ at before and after two week storage. 
Table I. Changes of Drug Contents and Peak Area Ratios Obtained from $S=O$ Stretching at $1094.1 \mathrm{~cm}^{-1}$ of Rabeprazole in Binary Blends with Pharmaceutical Excipients (3:1 w/W/ ratio) after Two Weeks of Storage Period

\begin{tabular}{cccc}
\hline \hline Component & Acidity/alkalinity & Content (\%) & Area ratio (\%)* \\
\hline Rabeprazole & - & $97.01 \pm 1.44$ & 91.04 \\
HPMC 4000 & $5.5-8.0$ & $101.33 \pm 0.64$ & 101.74 \\
MgO & 10.3 & $101.97 \pm 2.16$ & 102.47 \\
Carbomer 934 & $2.3-3.0$ & $56.75 \pm 2.00$ & 60.47 \\
\hline
\end{tabular}

*Area ratio (\%)was calculated by dividing the FTIR $\mathrm{S}=\mathrm{O}$ band area at $1094 \mathrm{~cm}^{-1}$ after two week storage relative to initial time.

blends with pharmaceutical excipients at a drug-excipient ratio of 3:1 before and after two weeks of storage. A band due to $\mathrm{S}=\mathrm{O}$ stretching can be observed at $1094.1 \mathrm{~cm}^{-1}$ in the FTIR spectrum of rabeprazole and this could be a stability indicator since its intensity decreases with the formation of thioether rabeprazole. It indicated the differences in $\mathrm{S}=\mathrm{O}$ stretching at $1094.1 \mathrm{~cm}^{-1}$ between drug and its binary blends before and after storage conditions. The corresponding $\mathrm{S}=\mathrm{O}$ peak area was also computed for comparison.

Table I shows the changes of drug contents and ratio of FTIR band areas of rabeprazole in binary blends with pharmaceutical excipients after two weeks of storage period. Drug contents significantly decreased in the presence of carbomer 934. The contents of rabeprazole alone also decreased during storage. In contrast, drug contents were almost unchanged in the presence of HPMC 4000 and MgO, indicating these excipients might act as stabilizers of rabeprazole. The relative $\mathrm{S}=\mathrm{O}$ peak area ratios also suggested that major rabeprazole decomposition occurred in the sample containing carbomer 934 .

\section{Effect of the drug-excipient ratios}

Solid-state reactions are generally heterogeneous reactions, which only occur at points of contact between drug and pharmaceutical excipients, and thus the drug-excipient ratios play an important role in a compatibility study (Serajuddin et al., 1999). Figure 4 illustrates the percentage of rabeprazole remaining in the presence of pharmaceutical excipients as a function of drug-excipient ratio after two weeks storage at $40^{\circ} \mathrm{C} / 75 \% \mathrm{RH}$. Rabeprazole appeared to be very stable with HPMC 4000 and $\mathrm{MgO}$ (alkalizer) at drug-excipient ratios. However, the stability of rabeprazole significantly decreased with the lowering ratio of drug-carbomer 934 from 3:1 to 1:3 in a dose dependent manner. The detailed information on degradation behaviors of rabeprazole and the formation of thioether rabeprazole in binary blends with carbomer 934 as a function of storage time at $40^{\circ} \mathrm{C}$ and $75 \% \mathrm{RH}$ are given in Fig-

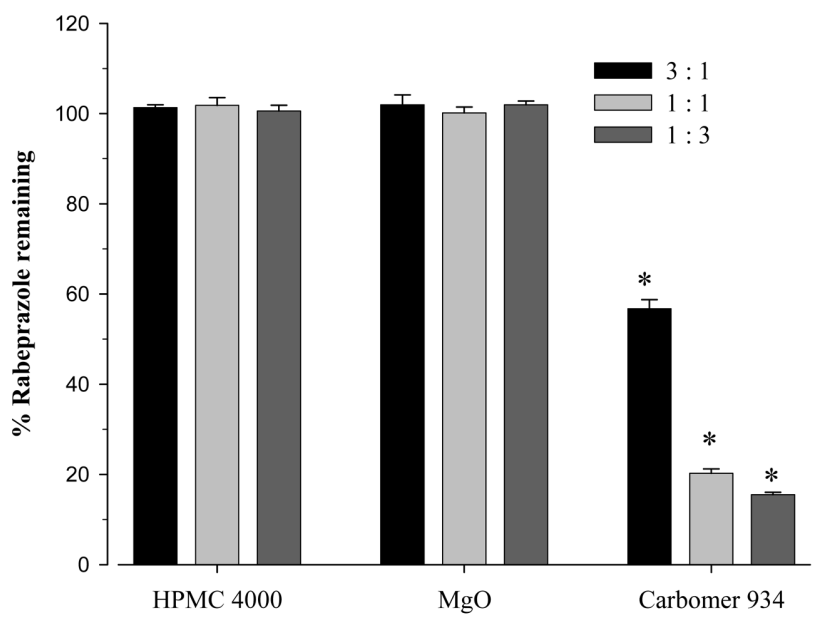

Figure 4. Percentage of rabeprazole remaining in the presence of pharmaceutical excipients as a function of drug-excipient ratio after two weeks of storage at $40^{\circ} \mathrm{C}$ and $75 \% \mathrm{RH}$. ${ }^{*} \mathrm{p}<0.05$, significantly different from HPLC 4000 or $\mathrm{MgO}$.

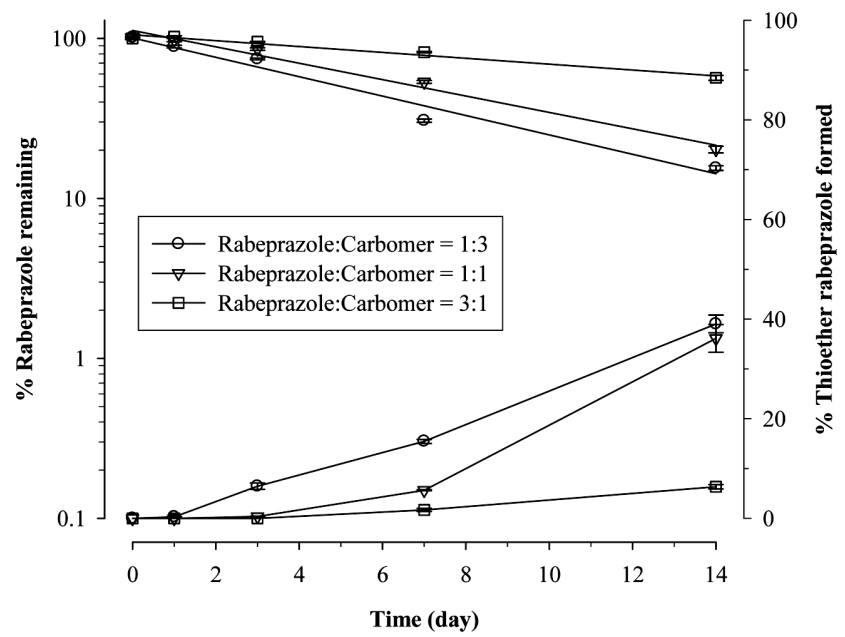

Figure 5. Degradation behaviors of rabeprazole and the formation of thioether rabeprazole in binary blends with carbomer 934 as a function of storage time at $40^{\circ} \mathrm{C}$ and $75 \% \mathrm{RH}$.

ure 5. Rabeprazole in binary blends with carbomer 934 was degraded more rapidly as a function of time. This kinetic degradation was more pronounced as the amount of carbomer 934 increased in a dose dependent manner. Table II summarizes the degradation kinetic parameters and peak area ratios obtained from $\mathrm{S}=\mathrm{O}$ stretching at $1094.1 \mathrm{~cm}^{-1}$ of rabeprazole in binary blends with carbomer 934 as a function of drug-excipient ratio. The kinetic parameters were generated by plotting the logarithm of percentage of rabeprazole remaining versus time by linear regression, assuming first-order kinetics of degradation. As the fraction of carbomer 934 in binary blend increased, the drug contents decreased, resulting in a shorter half-life. Furthermore, the relative peak area ratios obtained from $\mathrm{S}=\mathrm{O}$ 
Table II. Degradation Kinetic Parameters and Peak Area Ratios Obtained from S=O Stretching at $1094.1 \mathrm{~cm}^{-1}$ of Rabeprazole in Binary Blends with Carbomer 934 as a Function of Drug-excipient Ratio

\begin{tabular}{ccccc}
\hline \hline Drug:carbomer 934 & $\mathrm{k}\left(\right.$ day $\left.^{-1}\right)$ & $\mathrm{t}_{1 / 2}$ (day) & Content $(\%)$ & Area ratio $(\%)^{*}$ \\
\hline $3: 1$ & $0.042 \pm 0.003$ & $16.44 \pm 1.20$ & $56.75 \pm 2.00$ & 60.47 \\
$1: 1$ & $0.118 \pm 0.002$ & $5.87 \pm 0.10$ & $20.23 \pm 0.98$ & 36.53 \\
$1: 3$ & $0.140 \pm 0.002$ & $4.96 \pm 0.06$ & $15.48 \pm 0.54$ & trace \\
\hline
\end{tabular}

*Area ratio \% $\left(1094 \mathrm{~cm}^{-1}\right)$ can be calculated by dividing the FTIR S=O band area at $1094 \mathrm{~cm}^{-1}$ after two week storage relative to initial time before the storage and multiplying by 100 .

stretching at $1094.1 \mathrm{~cm}^{-1}$ consistently decreased as discussed previously.

Overall, rabeprazole is more compatible with neutral and alkaline pharmaceutical excipients like HPMC 4000 and $\mathrm{MgO}$, but is completely incompatible with pharmaceutical excipients possessing acidic functional groups like carbomer 934 . This can be explained by the sensitivity of the basic benzimidazole nitrogen in the structure of rabeprazole (Tutunji et al., 2006).

\section{Proposed degradation mechanism with carbomer}

Preclinical studies have demonstrated that rabeprazole is a protonable weak base with a highest ionization constant (approximately $\mathrm{pKa}=5.0$ ) and hence undergoes the most rapid degradation in acidic and neutral environments among all PPIs (Wong and Sung, 2006). Carbomer is an acrylic acid polymer (approximately $\mathrm{pH}=2.5-3.0$ ) and contains $56-68 \%$ carboxylic acid $(\mathrm{COOH})$ groups in structures on the dry basis (Rowe et al., 2003). It is also hydroscopic and its typical equilibrium moisture content at $25^{\circ} \mathrm{C} / 50 \% \mathrm{RH}$ is $8-10 \%$. At low $\mathrm{pH}$, the carboxylic acid groups of carbomer molecules are uncharged but it forms negatively charged carboxylates in near neutal or alkaline media (Bommareddy et al., 2006). Since the major degradation pathway for rabeprazole is acid-catalyzed, the drug decomposition in the binary blends can be attributed to the ionized carboxylic acid groups of carbomer 934, which can interact with basic benzimidazole nitrogen of rabeprazole (Tutunji et al., 2006). In contrast, $\mathrm{MgO}$ is basic oxide $(\mathrm{pKa}=10.3)$ in aqueous solution. However, it forms $\mathrm{Mg}(\mathrm{OH})_{2}$ due to its hygroscopicity in the air and as such can be expected to stabilize drug by producing a stable microenvironmental condition in the solid-state binary blends. Additionally, HPMC 4000 is a stable nonionic material. It has no reactive functional group to degrade the drug $\mathrm{S}=\mathrm{O}$ structure (see Figure 3), giving good compatibility between drug and HPMC 4000 in a solid state during storage conditions.

\section{Conclusions}

RPN was very compatible with neutral and alkaline phar- maceutical excipients like HPMC 4000 and MgO (alkalizer), but was completely incompatible with carbomer 934 possessing acidic functional groups due to its sensitivity of the basic benzimidazole nitrogen moiety of rabeprazole. A sensitive and reliable chromatographic analysis combined with other instrumental analyses like FTIR could be readily applicable to the quick evaluation of the solid-state compatibility and degradation mechanism of rabeprazole with various pharmaceutical excipients.

\section{Acknowledgements}

This work was supported by the Ministry of Education, Science and Technology and by a grant of the Korean Health Technology R\&D Project, Ministry for Health and Welfare (A092019). We also thank the Central Research laboratory for the use of FTIR and LC/MS, and the Research Institute of Pharmaceutical Sciences, Kangwon National University for allowing the use of their HPLC systems. Finally, we acknowledge KBSI for the use of SEM.

\section{References}

Bommareddy, G.S., Paker-Leggs, S., Saripella, K.K., Neau, S.H., 2006. Extruded and spheronized beads containing Carbopol 974P to deliver nonelectrolytes and salts of weakly basic drugs. Int. J. Pharm. 321, 62-71.

Bruni, G., Amici, L., Berbenni, V., Marini, A., Orlandi, A., 2002. Drug-excipient compatibility studies. Search of interaction indicators. J. Therm. Anal. Cal. 68, 561-573.

Byrn, S.R., Xu, W., Newman, A.W., 2001. Chemical reactivity in solid-state pharmaceuticals: formulation implications. Adv. Drug Deliv. Rev. 48, 115-136.

Carswell, C., Goa, K., 2001. Rabeprazole: An update of its use in acid-related disorders. Drugs 61, 2327-2356.

Cornaire, G., Woodley, J., Hermann, P., Cloarec, A., Arellano, C., Houin, G., 2004. Impact of excipients on the absorption of Pglycoprotein substrates in vitro and in vivo. Int. J. Pharm. 278, 119-131.

Desta, Z., Zhao, X., Shin, J.G., Flockhart, D.A., 2002. Clinical significance of the cytochrome $\mathrm{P} 4502 \mathrm{C} 19$ genetic polymor- 
phism. Clin. Pharmacokinet. 41, 913-958.

El-Gindy, A., El-Yazby, F., Maher, M.M., 2003. Spectrophotometric and chromatographic determination of rabeprazole in presence of its degradation products. J. Pharm. Biomed. Anal. 31, 229-242.

Fujioka, T., Kawasaki, H., Su, W.W., Nasu, M., 1993. In vitro anti microbial activity against $H$. pylori and clinical efficacy of various drugs. Jpn. J. Clin. Med. 51, 3255-3260.

Gaisford, S., 2005. Stability assessment of pharmaceuticals and biopharmaceuticals by isothermal calorimetry. Curr. Pharm. Biotechnol. 6, 181-191.

Huang, L.F., Tong, W.Q., 2004. Impact of solid state properties on developability assessment of drug candidates. Adv. Drug Deliv. Rev. 56, 321-334.

Karabas, I., Orkoula, M.G., Kontoyannis, C.G., 2007. Analysis and stability of polymorphs in tablets: the case of risperidone. Talanta $71,1382-1386$.

Loukas, Y.L., Vraka, V., Gregoriadis, G., 1998. Drugs, in cyclodextrins, in liposomes: a novel approach to the chemical stability of drugs sensitive to hydrolysis. Int. J. Pharm. 162, 137142.

Morii, M., Takata, H., Fujisaki, H., Takeguchi, N., 1990. The potency of substituted benzimidazoles such as E3810, omeprazole, RO 18-5364 to inhibit gastric $\mathrm{H}^{+}, \mathrm{K}^{+}$-ATPase is correlated with the rate of acid-activation of the inhibitor. Biochem. Pharmacol. 39, 661-667.

Neugebauer, H., Brabec, C.J., Hummelen, J.C., Janssen, R.A.J., Sariciftci, N.S., 1999. Stability studies and degradation analysis of plastic solar cell materials by FTIR spectroscopy. Synth. Metals 102, 1002-1003.

Richardson, P., Hawkey, C.J., Stack, W.A., 1998. Proton pump inhibitors: pharmacology and rationale for use in gastrointestinal disorders. Drugs 56, 307-335.

Rowe, R.C., Sheskey, P.J., Weller, P.J., 2003. Handbook of Pharmaceutical Excipients, $4^{\text {th }}$ ed. Pharmaceutical Press, London, UK.
Serajuddin, A.T., Thakur, A.B., Ghoshal, R.N., Fakes, M.G., Ranadive, S.A., Morris, K.R., Varia, S.A., 1999. Selection of solid dosage form composition through drug-excipient compatibility testing. J. Pharm. Sci. 88, 696-704.

Tutunji, M.F., Qaisi, A.M., El-Eswed, B., Tutunji, L.F., 2006. An in vitro investigation on acid catalyzed reactions of proton pump inhibitors in the absence of an electrophile. Int. J. Pharm. 323, 110-116.

Uno, T., Yasui-Furukori, N., Shimizu, M., Sugawara, K., Tateishi, T., 2005. Determination of rabeprazole and its active metabolite, rabeprazole thioether in human plasma by columnswitching high-performance liquid chromatography and its application to pharmacokinetic study. J. Chromatogr. B 824, 238-243.

Urbanetz, N.A., 2006. Stabilization of solid dispersions of nimodipine and polyethylene glycol 2000. Eur. J. Pharm. Sci. 28, 67-76.

Verma, R.K, Garg, S., 2005. Selection of excipients for extended release formulations of Glipizide through drug-excipient compatibility testing. J. Pharm. Biomed. Anal. 38, 633-644.

Wang, S.W., Monagle, J., McNulty, C., Putnam, D., Chen, H., 2004. Determination of P-glycoprotein inhibition by excipients and their combinations using an integrated high-throughput process. J. Pharm. Sci. 93, 2755-2767.

Waterman, K.C., Adami, R.C., 2005. Accelerated aging: Prediction of chemical stability of pharmaceuticals. Int. J. Pharm. 293, 101-125.

Wong, L.H.G., Sung, J.Y.J., 2006. Esomeprazole: a new proton pump inhibitor for NSAID-associated peptic ulcers and dyspepsia. Therapy 3, 227-236.

Wyttenbach, N., Birringer, C., Alsenz, J., Kuentz, M., 2005. Drugexcipient compatibility testing using a high-throughput approach and statistical design. Pharm. Dev. Technol. 10, 499-505.

Zhang, Y., Chen, X., Gu, Q., Zhong, D., 2004. Quantification of rabeprazole in human plasma by liquid chromatography-tandem mass spectrometry. Anal. Chim. Acta 523, 171-175. 